\title{
Probing the Native Structure and Chemistry of Dendrites and SEI Layers in Li- Metal Batteries by Cryo-FIB Lift-Out and Cryo-STEM
}

\author{
Michael J. Zachman ${ }^{1}$, Zhengyuan $\mathrm{Tu}^{2}$, Lynden A. Archer ${ }^{2,3}$, and Lena F. Kourkoutis ${ }^{1,4}$ \\ 1. School of Applied and Engineering Physics, Cornell University, Ithaca, NY 14853, USA. \\ 2. Department of Materials Science and Engineering, Cornell University, Ithaca, NY 14853, USA. \\ 3. Robert Frederick Smith School of Chemical and Biomolecular Engineering, Cornell University, \\ Ithaca, NY 14853, USA. \\ 4. Kavli Institute for Nanoscale Science, Cornell University, Ithaca, NY 14853, USA.
}

Processes at solid-liquid interfaces play a critical role in a range of chemical, physical, and biological systems. One example is the formation of dendrites during charging of lithium metal batteries (LMBs), which is closely tied to processes at the metal-electrode/liquid-electrolyte interface, including formation of a nanoscale solid-electrolyte interphase (SEI) layer [1]. The resulting dendritic growths lead to rapid capacity fade and safety concerns [2]. Therefore, despite their promise for large enhancements in energy density, rechargeable LMBs able to live up to this promise have been elusive.

The ability to directly probe the nanoscale structure and chemistry of solid-liquid interfaces would significantly aid our understanding of processes occurring in these systems, but accurate characterization is difficult due to the complexity and buried nature of these interfaces, necessitating the development of new analytical techniques [3]. Electrode-electrolyte interfaces in LMBs introduce additional challenges, such as the high chemical reactivity of lithium metal anodes and the volatility of commonly used liquid electrolytes. To address some of these issues, the liquid electrolyte is typically removed and the electrode washed and dried before being characterized by traditional methods, which alters the structure and chemistry of the solid-liquid interface.

Here, we rapidly freeze the liquid electrolytes to preserve the native structure and chemistry of the solidliquid interfaces and significantly reduce the reactivity of the materials present. By coupling analytic cryogenic scanning transmission electron microscopy (cryo-STEM) and cryogenic focused ion beam (cryo-FIB) lift-out [4], we then map the intact nanoscale interior structure and chemistry of the dendrites and their interfaces in electron transparent lamellas taken from LMBs (Fig. 1). Avoiding the washing steps allows us to discover that two distinct families of dendrites coexist on lithium anodes in these batteries. While lithium dendrites are generally assumed to be composed principally of metal, analysis of the dendrites' Li-K edge fine structure allows us to demonstrate the presence of lithium hydride dendrites, which may contribute disproportionately to battery capacity fade and failure. Plasmon mapping reveals the distribution of materials within the dendrites (Fig. 2), which allows us to propose a formation mechanism of lithium hydride dendrites [5].

[1] X.-B. Cheng et al., Adv. Sci. 3 (2016), p. 1500213.

[2] J.-M. Tarascon and M. Armand, Nature 414 (2001), p. 359.

[3] F. Zaera, Chem. Rev. 112 (2012), p. 2920.

[4] M. Zachman et al., Microsc. Microanal. 22 (2016), p. 1338.

[5] This work was supported by NSF (DMR-1654596, DMR-1429155, DMR-1719875) and the Packard Foundation. 

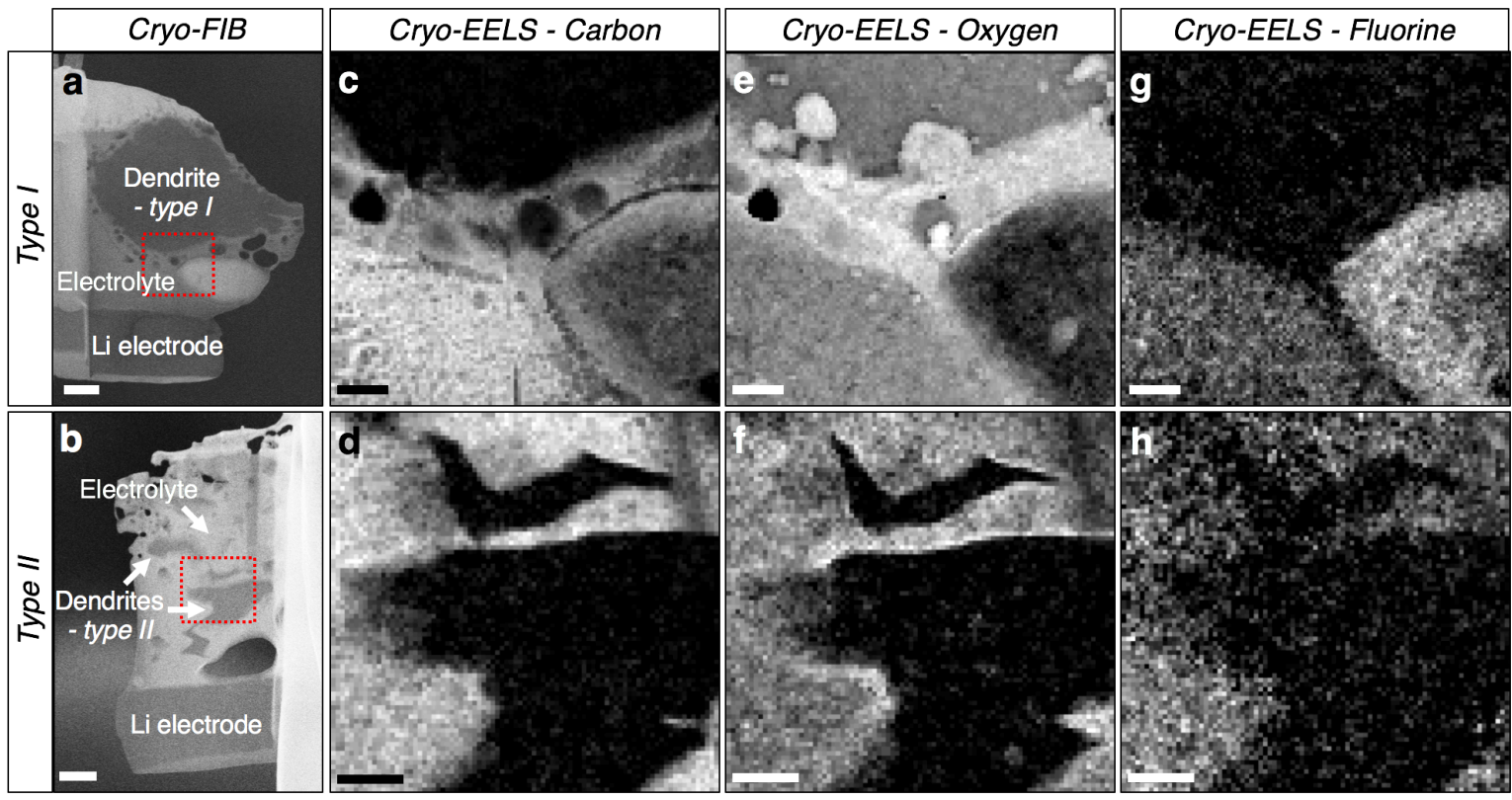

Figure 1. Elemental composition of dendrites and their associated interphase layers probed by cryoSTEM-EELS. ( $a, b)$ Electron transparent lamellas of both dendrite types, produced by cryo-FIB lift-out. (c-h) EELS elemental maps show an extended oxygen-rich SEI on the type I dendrite, and a thin type II SEI containing oxygen, but no carbon. The type I dendrite had an appreciable oxygen content, while the type II did not. Fluorine-rich structures were often observed near both dendrite types. The small bubbles present in the electrolyte are likely due to hydrogen liberation from beam exposure, while the large bubbles in the type I SEI are inherent to the structure. (a, b) scale bars, $1 \mu \mathrm{m},(\mathrm{c}-\mathrm{h}), 300 \mathrm{~nm}$.
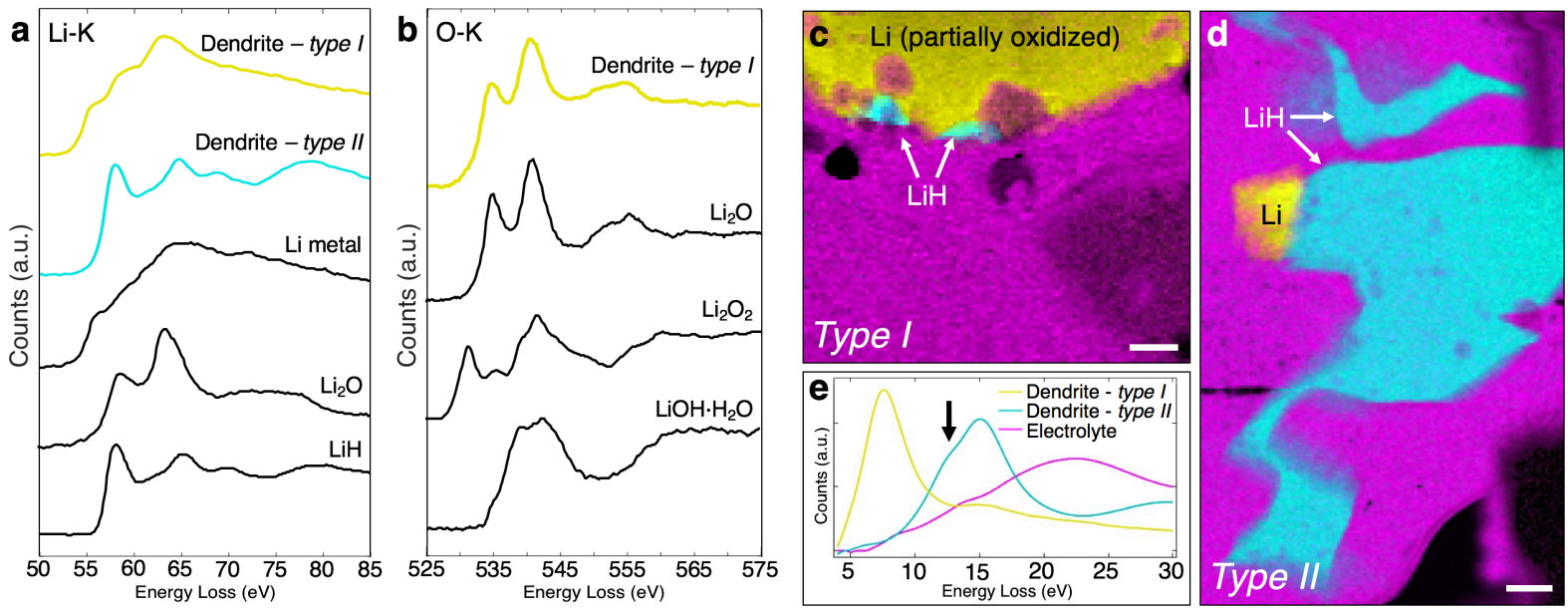

Figure 2. Comparison of dendrite core-loss EELS fine structure with reference materials and compositional mapping by low-loss EELS ( $a, b)$ Comparison of the dendrites' lithium and oxygen K-edge fine structures with reference materials reveals that the type I dendrite is composed of partially oxidized lithium metal while the type II dendrite is uniform lithium hydride. (c, d) Mapping of the distinct lithium metal and hydride low-loss EELS spectra (e) demonstrates that the type I dendrite is only slightly oxidized and has small regions of $\mathrm{LiH}$ at its surface, while the type II dendrite has a lithium particle at its tip. The hydrogen K-edge appears as a shoulder at $\sim 13 \mathrm{eV}$ in the type II dendrite, denoted by the arrow. Scale bars, $300 \mathrm{~nm}$. 